\title{
On the Arithmetic of Abelian Varieties *
}

\author{
J.S. Milne (London)
}

In $\S 1$ we consider the situation: $L / K$ is a finite separable field extension, $A$ is an abelian variety over $L$, and $A_{*}$ is the abelian variety over $K$ obtained from $A$ by restriction of scalars. We study the arithmetic properties of $A_{*}$ relative to those of $A$, and in particular show that the conjectures of Birch and Swinnerton-Dyer hold for $A$ if and only if they hold for $A_{*}$.

In $\S 2$ we study certain twisted products of abelian varieties and use our results to show that the conjectures of Birch and Swinnerton-Dyer are true for a large class of twisted constant elliptic curves over function fields.

In $\S 3$ we develop a method of handling abelian varieties over a number field $K$ which are of $C M$-type but which do not have all their complex multiplications defined over $K$. In particular we compute under quite general conditions the conductors and zeta functions of such abelian varieties and so verify Serre's conjecture [12] on the form of the functional equation. Similar, but less complete, results have been obtained by Deuring [1] for elliptic curves and Shimura [15] for abelian varieties.

\section{\$1. The Arithmetic Invariants of the Norm}

Let $T \rightarrow S$ be a morphism of schemes. We recall the definition and properties of the norm functor $N_{T / S}$ (in [19] this is denoted by $R_{T / S}$ and called restriction of field of definition, and in [3, Exp. 195] it is denoted by $\Pi_{T / S}$ ). If $X$ is a $T$-scheme then $N_{T / S} X$ is uniquely determined as the $S$-scheme which represents the functor on $S$-schemes $Z \mapsto X\left(Z_{T}\right)$, where $Z_{T}=Z \times{ }_{S} T$. There is a $T$-morphism $p:\left(N_{T / S} X\right)_{T} \rightarrow X$ such that any other $T$-morphism $p^{\prime}: Z_{T} \rightarrow X$ factors uniquely as $p^{\prime}=p q_{T}$ with $q: Z \rightarrow N_{T / S} X$ an $S$-morphism. $N_{T S} X$ always exists if $X$ is quasi-projective and $T \rightarrow S$ is finite and faithfully flat [3, Exp. 221], and it is obvious from the definition that $N_{T S S}$ commutes with base change on $S$. If $X$ is a group scheme then $N_{T / S} X$ acquires a unique group structure such that $p$ is a morphism of group schemes. If $X$ is smooth over $T$ then it is obvious from the

* This research was supported by the Science Research Council of Great Britain.

13 Inventiones math., Vol. 17 
functorial definition of smoothness [4, IV] that $N_{T / S} X$ is smooth. If $X$ is an abelian scheme then $N_{T / S} X$ need not be an abelian scheme even (as Mumford has observed) if $T \rightarrow S$ corresponds to a finite field extension $L / K$. Indeed, if $L / K$ is purely inseparable of degree $m$ and $A$ is an abelian variety of dimension $d$ over $L$, then $L \otimes_{K} L=R$ is a local Artin ring with residue field $L$ and $N_{R / L} A_{R}=\left(N_{L / K} A\right) \otimes_{K} L$ is an extension of $A$ by a unipotent group scheme group scheme of dimension $(m-1) d$ [2, p. 263]. However if $L / K$ is separable then $N_{L / K} A$ is an abelian variety because, for any Galois extensions $\bar{K}$ of $K$ containing $L$, there is an isomorphism $P:\left(N_{L / K} A\right)_{K} \rightarrow A_{K}^{\sigma_{1}} \times \cdots \times A_{R}^{\sigma_{m}}$ where $\sigma_{1}, \ldots, \sigma_{m}$ are the distinct embeddings of $L$ in $\bar{K}$ over $K\left[19\right.$, p. 5], and so $\left(N_{L / K} A\right)_{K}$ is an abelian variety.

For the remainder of this section $L / K$ will be a finite separable field extension of degree $m, A$ an abelian variety over $L$ of dimension $d, \bar{K}$ a Galois extension of $K$ containing $L$ (often equal to a separable algebraic closure $K_{s}$ of $\left.K\right), G=\operatorname{Gal}(\bar{K} / K), H=\operatorname{Gal}(\bar{K} / L)$, and $\left\{\sigma_{1}, \ldots, \sigma_{m}\right\}$ a set of left coset representatives for $H$ in $G$. We will compute the arithmetic invariants of $A_{*}=N_{L / K} A$.

(a) Points. $A_{*}(K)=A(L)$ and so their ranks (if finite) are equal. The morphism $P$ above induces an isomorphism $A_{*}(\bar{K}) \rightarrow \mathrm{Z}[G] \otimes_{\mathbf{Z}[H]} A(\bar{K})$ and this, with $\bar{K}=K_{s}$, induces canonical isomorphisms

$$
T_{l} A_{*} \approx \mathbf{Z}_{l}[G] \otimes_{\mathbf{Z}_{l}[H]} T_{l} A \text { and } V_{l} A_{*} \approx \mathbf{Q}_{l}[G] \otimes_{\mathbf{Q}_{l}[H]} V_{l} A
$$

In other words, the $l$-adic representation of $G$ on $T_{l} A_{*}$ (resp. $V_{l} A_{*}$ ) is the induced representation coming from the representation of $H$ on $T_{l} A$ (resp. $\left.V_{l} A\right)$.

(b) Conductors. Let $L$ be the field of fractions of a complete discrete valuation ring with finite residue field, and let $V$ be a finite dimensional vector space over $\mathbf{Q}_{l}$ where $l$ is not equal to the residue characteristic of $L$. Take $\bar{K}=K_{\mathrm{s}}$ and let $\rho$ be an $l$-adic representation of $H$ on $V . \rho$ automatically satisfies condition $\left(H_{\rho}\right)$ of [12] and so the exponent of the tame conductor $\varepsilon(\rho)$ (resp. wild conductor $\delta(\rho)$, resp. conductor $f(\rho)=$ $\varepsilon(\rho)+\delta(\rho))$ is defined. See [12] for the details. Then

Lemma. Let $\rho_{*}$ be the representation of $G=\operatorname{Gal}\left(K_{s} / K\right)$ induced by $\rho$.

$$
\begin{aligned}
& \varepsilon\left(\rho_{*}\right)=\varepsilon(\rho)+(m-1) \operatorname{dim}(V), \\
& \delta\left(\rho_{*}\right)=\delta(\rho)+(\beta-m+1) \operatorname{dim}(V), \\
& f\left(\rho_{*}\right)=f(\rho)+\beta \operatorname{dim}(V)
\end{aligned}
$$

where $\beta$ is the exponent of the discriminant of $L / K$.

Proof. Straightforward using [11, VI Proposition 4]. 
When $\rho_{l}$ is the representation of $H$ defined by $V_{l} A$, Grothendieck [5] has shown that $\delta\left(\rho_{l}\right)$ is independent of $l$ (different from the residue characteristic). $\varepsilon\left(\rho_{l}\right)$ is obviously independent of $l$ because it equals $\mu(A)+2 \lambda(A)$ where $\mu(A)$ and $\lambda(A)$ are the dimensions of the reductive and unipotent parts of the reduction of $A$. Thus, there are numbers $\varepsilon(A), \delta(A), f(A)$ depending only on $A$ over $L$.

Now take $L$ to be a global field i.e. a number field or function field in one variable over a finite field. In multiplicative notation, the conductor of $A$ is the ideal or divisor $f(A)=\prod_{w} \mathfrak{p}_{w}^{f(w)}$ where $w$ runs through the non-archimedean primes of $L, L_{w}$ is the completion of $L$ at $w$, and $f(w)=f\left(A_{L_{w}}\right)$.

Proposition 1. With the above notations, $\mathfrak{f}\left(A_{*}\right)=N_{L / K}(\mathfrak{f}(A)) d_{L / K}^{2 d}$, where here $N_{L / K}$ refers to taking norms of ideals or divisors, and $d_{L / K}$ is the discriminant of $L$ over $K$. In particular, $A_{*}$ has good reduction at $v$ if and only if $v$ does not divide the discriminant of Lover $K$ and $A$ has good reduction at all primes of $L$ dividings $v$.

Proof. Immediate from the lemma.

Remark. Let $L / K$ be an extension of local fields with ramification index $e$, and let $\alpha(A)$ be the dimension of the part of the reduction of $A$ which is an abelian variety.

Then

$$
\begin{aligned}
\alpha\left(A_{*}\right) & =\frac{m}{e} \alpha(A), \\
\mu\left(A_{*}\right) & =\frac{m}{e} \mu(A), \\
\lambda\left(A_{*}\right) & =\frac{m}{e}(d e-d+\lambda(A)) .
\end{aligned}
$$

Indeed, if $e=1$ this is obvious by looking at the norm of the Néron minimal model of $A$ (see the next section (c)). If $e=m$ it follows from the formula $\varepsilon\left(A_{*}\right)=\varepsilon(A)+(m-1) 2 d$ and the obvious facts that $\alpha\left(A_{*}\right) \geqq \alpha(A)$, $\mu\left(A_{*}\right) \geqq \mu(A)$ (obvious, because $p: A_{* L} \rightarrow A$ is surjective). The general case follows by transitivity.

If $L$ is a number field, write $\left.d_{L}=\mid d_{L / Q}\right\}$, and if $L$ is a function field in one variable over a finite field with $q$ elements, write $d_{L}=q^{2 g-2}$ where $\mathrm{g}$ is the genus of $L$. Define $N_{L}(\mathfrak{f}(A))=\prod_{w} N w^{f(w)}$ where $w$ runs through the non-archimedean primes of $L$ and $N w$ is the number of elements of the residue field $k(w)$ at $w$. Finally define $c(A)=N_{L}(\mathfrak{f}(A)) d_{L}^{2 \operatorname{dim}(A)}[12$, p. 12]. $13^{*}$ 
Corollary $c\left(A_{*}\right)=c(A)$.

Proof. Immediate from the theorem, the formula for the transitivity of norms, and the Hurwitz genus formula.

(c) Tamagawa Numbers. $L$ is a global field. Let $\omega$ be a non-zero invariant exterior differential form of degree $d$ on $A$. Define $\lambda_{w}=1$ if $w$ is archimedean, and $\lambda_{w}=\frac{(N w)^{d}}{n_{w}}$ where $n_{w}$ is the order of $A_{w, 0}^{0}(k(w))$, the group of points on the connected component of zero of the reduction of the Néron minimal model of $A$, if $w$ is non-archimedean. By $[19,2.2 .5]$ the $\lambda_{w}$ form a set of convergence factors for $A$. We define $\tau(A)$ to be the measure of the adèle group of $A$ relative to the Tamagawa measure $\Omega=\left(\omega,\left(\lambda_{w}\right)\right)[19$, p. 23$]$.

Let $\omega_{*}$ be the invariant exterior differential form on $A_{*}$ corresponding to $\omega$ as in $[19$, p. 24$]$. Proposition 2. (a) $\lambda_{v}=\prod_{w / v} \lambda_{w}$ is equal to $\frac{(N v)^{\operatorname{dim}\left(A_{*}\right)}}{n_{v}}$ for any non-
archimedean prime $v$ of $K$.

(b) $\tau(A)=\tau\left(A_{*}\right)$.

Proof. (a) Let $A_{w}$ be the Néron minimal model of $A$ over $R_{w}$, the completion of the integers of $L$ at w. $A_{w}$ is quasi-projective and so $A_{w_{*} *}=$ $N_{R_{w} / R_{v}} A_{w}$ exists. Clearly $A_{w *} \approx A_{* v}$, the Néron minimal model of $A_{*}$, because it is a smooth group scheme with the correct functorial property. Moreover the zero component $A_{* v}^{0}$ of $A_{* r}$ is isomorphic to $\left(A_{w}^{0}\right)_{*}$ because $\left(A_{w}^{0}\right)_{*}$ is an open subgroup scheme of $A_{w *}$ with connected fibres.

If $R_{w}$ is unramified over $R_{v}$, then $A_{* v}^{0} \otimes_{R_{v}} k(v) \approx N_{k(w) / k(v)}\left(A_{w}^{0} \otimes_{R_{w}} k(w)\right)$ and so $n_{w}=n_{v}, N w=N v^{m^{\prime}}$, and $\frac{N w^{d}}{n_{w}}=\frac{N v^{m^{\prime} d}}{n_{v}}$, where $m^{\prime}=\left[R_{w^{\prime}}: R_{v}\right]$.

If $R_{w}$ is totally ramified over $R_{v}$, then $A_{* v}^{0} \otimes_{R_{v}} k(v) \approx$ $N_{R_{w, m^{\prime} / k}}\left(A_{w}^{0} \otimes_{R_{w}} R_{w, m^{\prime}}\right)$ where $R_{w, m^{\prime}}$ is $R_{w}$ modulo the $m^{\prime}$ th power of its maximal ideal. Thus $n_{v}=\operatorname{order}$ of $A_{w}^{0}\left(R_{w, m^{\prime}}\right)=N v^{\left(m^{\prime}-1\right) d} n_{w}$ because $A_{w}^{0}$ is smooth. $N v=N w$ and so $\frac{N w^{d}}{n_{w}}=\frac{N v^{m^{\prime} d}}{n^{v}}$, and this suffices to complete
the proof.

(b) Follows from (a) and [19, 2.3.2].

(d) Zeta Functions. $L$ is again a global field. For any non-archimedean prime $w$ of $L$ we write $I_{w}$ for an inertia group of $w$ and $\pi_{w}$ for a Frobenius element of $H / I_{w}$. Following [12] we define, for any prime $l \neq \operatorname{char}(k(w))$, a polynomial $P_{A, w}(T)=\operatorname{det}\left(1-T \pi_{w}\right)$ where $\pi_{w}$ is regarded as acting on $\left(V_{l} A\right)^{I_{w}}=V_{l}\left(A_{w}^{0} \otimes_{R_{w}} k(w)\right)$. Conjectures $C_{5}, C_{6}, C_{7}$ (loc. cit.) are known to be true in this case. Define 
$\zeta_{A}(s)=\prod_{w} P_{A, w}\left(N w^{-s}\right)^{-1}, \quad \zeta_{A}^{*}(s)=\frac{\zeta_{A}(s)}{\tau(A)}, \quad \xi_{A}(s)=c(A)^{s / 2}\left(\frac{\Gamma(s)}{(2 \pi)^{+s}}\right)^{n d} \zeta_{A}(s)$ where $n=0$ if $L$ is a function field and $n=[L: \mathbf{Q}]$ if $L$ is number field.

Proposition 3. $\zeta_{A_{*}}(s)=\zeta_{A}(s), \zeta_{A_{*}}^{*}(s)=\zeta_{A}^{*}(s), \xi_{A_{*}}(s)=\zeta_{A}(s)$.

Proof. After (b) and (c) it suffices to prove the first statement, and for this it suffices to show that $\prod_{w \mid x} P_{A, w}\left(N w^{-s}\right)=P_{A_{*}, v}\left(N v^{-s}\right)$. By passing to the completions, we may assume that $w$ is the only prime of $L$ lying over $v$. If $L / K$ is unramified at $v$, then $\left(V_{l} A_{*}\right)^{I_{v}}=\mathbf{Q}_{l}[G / H] \otimes\left(V_{l} A\right)^{I_{\mathrm{ww}}}$, and $G / H$ is a finite cyclic group of order $m$ generated by the class of $\pi_{t}$. It follows that $P_{A_{*},}(T)=P_{A, w}\left(T^{m}\right)$, which gives the required equality. If $L / K$ is totally ramified at $v$, then $\left(V_{l} A_{*}\right)^{I_{v}}=\left(V_{l} A\right)^{I_{w}}, \pi_{v}=\pi_{w}$, and the result is obvious.

Remark. Consider any projective smooth scheme $V$ over $L$ and let $V_{*}=N_{L / K} V$. Then it is possible to prove as above that

$$
\zeta_{V_{*}}(s)=\zeta_{V}(s), \quad c\left(V_{*}\right)=c(V), \quad \xi_{V_{*}}(s)=\zeta_{V}(s)
$$

Indeed, $H^{1}\left(\bar{V}_{*}, \mathbf{Q}_{l}\right) \approx \mathbf{Q}_{l}[G] \otimes_{\mathbf{Q}_{l}[H]} H^{1}\left(\bar{V}, \mathbf{Q}_{l}\right)$, because

$$
H^{1}\left(\vec{V}, \mathbf{Q}_{l}\right) \otimes_{\mathbf{Q}_{l}} V_{l} \mathbf{G}_{m} \approx V_{l} B,
$$

where $B$ is the Picard variety of $V$, and $\operatorname{Pic}^{0}\left(V_{*}\right)$ can be computed as in (e) below. (Note that $V_{l} A=\operatorname{Hom}_{\mathbf{Q}_{l}}\left(H^{1}\left(\bar{A}, \mathbf{Q}_{l}\right), \mathbf{Q}_{l}\right)$ so that we have actually been working with the dual of $H^{1}\left(\bar{A}, \mathbf{Q}_{l}\right)$ rather than with $H^{1}\left(\bar{A}, \mathbf{Q}_{l}\right)$ itself. However, this affects nothing.) The first two equalities follow immediately from the isomorphism as above. The only additional point for the last equality is to check that the $I$-factors agree, but this is easy.

(e) $\mathrm{Pic}^{0}$. Let $b \in \operatorname{Pic}^{0}(A)$. The element $p^{\sigma_{1} *}\left(b^{\sigma_{1}}\right)+\cdots+p^{\sigma_{m} *}\left(b^{\sigma_{m}}\right)$ of $\mathrm{Pic}^{\circ}\left(A_{* \mathrm{R}}\right)$ is fixed under the action of $G$ and so determines an element $b_{*}$ of $\operatorname{Pic}^{0}\left(A_{*}\right)$.

Proposition 4. The map $b \mapsto b_{*}$ is an isomorphism $\operatorname{Pic}^{0}(A) \rightarrow \operatorname{Pic}^{0}\left(A_{*}\right)$.

Proof. This follows easily from the fact that $A \mapsto \operatorname{Pic}^{\circ}(A)$ is an additive functor on the category of abelian varieties over $L[8, \mathrm{p} .75]$ and so commutes with products.

(f) Heights. $L$ is a global field. We refer to $[16$, p. 5$]$ for the definition of the logarithmic height pairing $\langle,\rangle_{L}: \mathrm{Pic}^{0}(A) \times A(L) \rightarrow \mathbf{R}$.

Proposition 5. Let $a \in A_{*}(K)$ and $b \in \operatorname{Pic}^{\circ}(A)$. Then

$$
\left\langle b_{*}, a\right\rangle_{K}=\langle b, p(a)\rangle_{L}
$$


Proof. Choose $\bar{K}$ to be finite over $K$, of degree $n$ say. Then, by using some obvious functorial properties of the height pairing, one gets that

$$
\begin{aligned}
\left\langle b_{*}, a\right\rangle_{K}=\frac{1}{n}\left\langle b_{*}, a\right\rangle_{\mathrm{K}} & =\frac{1}{n} \sum_{j=1}^{m}\left\langle p^{\sigma_{J} *}\left(b^{\sigma_{J}}\right), a\right\rangle_{K} \\
& =\frac{1}{n} \sum_{j=1}^{m}\left\langle b^{\sigma_{J}}, p^{\sigma_{j}}(a)\right\rangle_{\mathrm{K}} \\
& =\frac{m}{n}\langle b, p(a)\rangle_{K} \\
& =\langle b, p(a)\rangle_{L} .
\end{aligned}
$$

Corollary. Let $\left\{a_{1}, \ldots, a_{r}\right\}$ (resp. $\left.\left\{b_{1}, \ldots, b_{r}\right\}\right)$ be a basis for $A_{*}(K)$ (resp. $\left.\operatorname{Pic}^{0}(A)\right)$ modulo torsion. Then $\left\{p\left(a_{1}\right), \ldots, p\left(a_{r}\right)\right\}\left(\right.$ resp. $\left.\left\{b_{1 *}, \ldots, b_{r *}\right\}\right)$ is a basis for $A(L)\left(\operatorname{resp} . \operatorname{Pic}^{0}\left(A_{*}\right)\right)$ modulo torsion, and

$$
\operatorname{det}\left(\left\langle b_{j *}, a_{i}\right\rangle\right)=\operatorname{det}\left(\left\langle b_{j}, p\left(a_{i}\right)\right\rangle\right) .
$$

We now apply the above to the conjectures of Birch and SwinnertonDyer. These state that,

$$
(B-S / D) \zeta_{A}^{*}(s) \sim \frac{[\mathrm{III}]\left|\operatorname{det}\left(\left\langle b_{i}, a_{\mathrm{j}}\right\rangle\right)\right|}{\left[A(K)_{\mathrm{tors}}\right]\left[A^{\prime}(K)_{\mathrm{tors}}\right]}(s-1)^{r}, \quad \text { as } s \rightarrow 1,
$$

where the symbols are as defined above or as defined in $[16, \S 1]$. For the sake of consistency, we must show that $\zeta_{A}^{*}(s) / L^{*}(s) \rightarrow 1$ as $s \rightarrow 1$, but this is a consequence of the following lemma.

Lemma. Let $M$ be a connected smooth commutative group scheme over a finite field $k$. If $P_{M}(T)=\operatorname{det}(1-\pi T)$ where $\pi$ is the Frobenius endomorphism regarded as acting on $V_{l} M, l \neq \operatorname{char}(k)$, then $P_{M}\left(q^{-1}\right)=\frac{[M(k)]}{q^{d}}$
where $q=[k]$ and $d=$ dimension of $M$.

Proof. If $0 \rightarrow M^{\prime} \rightarrow M \rightarrow M^{\prime \prime} \rightarrow 0$ is an exact sequence of group schemes then $P_{M}(T)=P_{M^{\prime}}(T) P_{M^{\prime \prime}}(T)$ and $[M(k)]=\left[M^{\prime}(k)\right]\left[M^{\prime \prime}(k)\right]$ (because $\left.H^{1}\left(k, M^{\prime}\right)=0\right)$. It follows that we need only prove the lemma for $M$ equal to an abelian variety, a unipotent group, or a torus. The first case is well-known. If $M=\mathbf{G}_{a}$, then $P_{M}=1$ and $[M(k)]=q$. The result follows for any unipotent $M$ because such a group has a composition series whose quotients are all isomorphic to $\mathbf{G}_{\boldsymbol{a}}$.

Finally, let $M$ be a torus. $P_{M}(T)=\operatorname{det}(1-T \hat{\pi})$ where $\hat{\pi}$ is $\pi$ regarded as acting on the character group $\hat{M}$ of $M$. Then $P_{M}\left(q^{-1}\right)=q^{-d} \operatorname{det}(q-\hat{\pi})=$ $q^{-d}[M(k)]$ (see [9]).

Theorem 1. $(B-S / D)$ is true for $A$ if and only if it is true for $A_{*}$. 
Proof. After the above, we know that all corresponding factors, except the Tate-Safarevič groups, are equal, but it is trivial to show that $\amalg(A) \approx \amalg\left(A_{*}\right)$ using $(a)$.

Corollary. Let Lbe a global field which is of degree $m$ over the rational number field or a rational function field $K_{0} .(B-S / D)$ is true for all abelian varieties of dimension $\leqq d$ over $L$ if it is true for all abelian varieties of dimension $\leqq m d$ orer $K_{0}$.

\section{§ 2. Forms of Products of Abelian Varieties}

Throughout this section, $\bar{K} / K$ will be a Galois extension with Galois group $G$, and $A$ an abelian variety of dimension $d$ over $K$. A $\bar{K} / K$-form of $A$ is a pair $\left(A^{\prime}, \psi\right)$ where $A^{\prime}$ is an abelian variety over $K$ and $\psi$ is an isomorphism $A_{\mathbb{K}} \rightarrow A_{\mathrm{K}}^{\prime}$. Then the map $\sigma \mapsto \psi^{-1} \psi^{\sigma}: G \rightarrow \operatorname{Aut}_{K}(A)$ is a 1 -cocycle for $G$ with values in $\operatorname{Aut}_{K}(A)$, and this correspondence sets up a bijection between the set of isomorphism classes of $\bar{K} / K$-forms of $A$ and the elements of $H^{1}\left(G\right.$, Aut $\left._{K}(A)\right)\left(G\right.$ acts on $A_{K}$ through its action on $\bar{K}$, and it acts on $\operatorname{Aut}_{K}(A)$ by $\left.\phi \mapsto \phi^{\sigma}=\sigma \phi \sigma^{-1}\right)$.

Let $R$ be a commutative subring of $\operatorname{End}_{K}(A)$ and let $M$ be an $R$-module, with a given isomorphism $\psi: R^{n} \rightarrow M$, on which $G$ acts (through a finite quotient if $\bar{K} / K$ is infinite). Then $\sigma \mapsto s(\sigma)=\psi^{-1} \psi^{\sigma}$ is a homomorphism $G \rightarrow G L_{n}(R)$ which may be regarded as a 1-cocycle for $G$. If $G L_{n}(R)$ is regarded as a subgroup of $\operatorname{Aut}_{K}\left(A^{n}\right)$ then we define $\left(M \otimes_{R} A, \psi_{A}\right)$ to be the $\bar{K} / K$-form of $A^{n}$ corresponding s. $(M, \psi) \mapsto\left(M \otimes_{R} A, \psi_{A}\right)$ can be extended to an additive functor; given $\phi: M \rightarrow M^{\prime}, \phi_{A}: M \otimes_{R} A \rightarrow M^{\prime} \otimes_{R} A$ is the homomorphism such that $\psi_{A}^{-1} \phi_{A K} \psi_{A}$ has the same matrix representation as $\psi^{-1} \phi \psi$.

If $\bar{K} / K$ is finite, then $R[G] \otimes_{R} A$ is isomorphic to $N_{K / K} A$.

Proposition 6. (a) If $\phi: M \rightarrow M^{\prime}$ has non-zero determinant $\operatorname{det}_{R}(\phi)$ with respect to the bases provided by $\psi$ and $\psi^{\prime}$, then $\phi_{A}$ is an isogeny of degree $\left|N_{R / \mathbf{Z}}\left(\operatorname{det}_{R}(\phi)\right)\right|^{2 d / r}$ where $r=\operatorname{rank}_{\mathbf{Z}}(R)$.

(b) $\psi_{A}$ induces isomorphisms of $G$-modules $M \otimes_{R} A(\bar{K}) \stackrel{\approx}{\longrightarrow}\left(M \otimes_{R} A\right)(\bar{K})$, $M \otimes_{R} T_{l} A \stackrel{\approx}{\rightrightarrows} T_{l}\left(M \otimes_{R} A\right), M \otimes_{R} V_{l} A \stackrel{\approx}{\rightrightarrows} V_{l}\left(M \otimes_{R} A\right)$.

(c) Let $K$ be a global field. Then

$$
\begin{aligned}
& \mathfrak{f}\left(M \otimes_{R} A\right)=\mathfrak{f}(M)^{2 d} \mathfrak{f}(A)^{n}, \\
& c\left(M \otimes_{R} A\right)=c(M)^{2 d} c(A)^{n}
\end{aligned}
$$

where $\mathfrak{j}$ and $c$ are the conductor and absolute conductor of $A$ or the character of the representation of $G$ on $M[11, \mathrm{VI}]$, provided $\mathfrak{f}(M)$ and $\mathfrak{f}(A)$ (resp. $c(M)$ and $c(A))$ have disjoint supports.

Proof. (a) Let $F$ be the field of fractions of $R$. Since field extension does not change degrees or determinants we may assume that $K=\bar{K}$. 
Then $M_{n}(F)$ is a simple Q-algebra and so, by [8, p. 179] it suffices to check that $\operatorname{deg} \phi_{A}=\left|N_{\mathrm{F} / \mathbf{Q}} \operatorname{det}_{R}(\phi)\right|^{2 d / r}$ for $\phi \in \mathbf{Z}$, but this is obvious.

(b) Follows directly from the definition of $M \otimes_{R} A$.

(c) Follows from (b) (cf. § 1).

Remark.1. The first isomorphism in (b) can be used to give a more invariant definition of $M \otimes_{R} A$.

2. It is possible to deduce the zeta function of $M \otimes_{R} A$ from that of $A$ and the representation of $G$ on $M$.

Example. Let $A$ be an abelian curve over $K$. Assume first that $j(A) \neq 0$, 1728 and that $\operatorname{char}(K) \neq 2$. Then $\operatorname{Aut}_{K_{s}}(A)=\operatorname{Aut}_{K}(A)=\{ \pm 1\}$ and $H^{1}\left(\operatorname{Gal}\left(K_{s} / K\right), \operatorname{Aut}_{K_{s}}(A)\right)=K^{*} / K^{* 2}$ by Kummer theory. Let $A_{d}$ be the $K_{\mathrm{s}} / K$-form of $A$ corresponding to $d \in K^{*}$. If $A$ has equation

$$
Y^{2}=X^{3}+a X^{2}+b X+c
$$

then $A_{d}$ has equation $d Y^{2}=X^{3}+a X^{2}+b X+c$ and $\psi$ is the map $(x, y) \mapsto(x, \sqrt{d} y)$. If $\bar{K}=K(\sqrt{d})$, then $A_{d}=\mathbf{Z}_{d} \otimes_{\mathbf{Z}} A$ where $\mathbf{Z}_{d}$ is $\mathbf{Z}$ with $\sigma \in G$ acting as 1 or -1 according as $\sigma$ is the identity or not. Thus if $K$ is a global field and $A$ has good reduction at a prime $v$ then $A_{d}$ has good reduction at $v$ if and only if $v$ is unramified in $K / K$. Moreover

$$
\zeta_{A_{d}}(s)=\prod_{v} \frac{1}{P_{A, v}((d / v)} \overline{\left.N v^{-s}\right)}
$$

(up to a finite number of factors) where $(d / v)$ is the quadratic residue symbol for $K$.

If $j(A) \neq 0$ but $\operatorname{char}(K)=2$, then $\operatorname{Aut}_{K_{s}}(A)=\operatorname{Aut}_{K}(A)=\{ \pm 1\}$, $H^{1}\left(\mathrm{Gal}\left(K_{s} / K\right), \operatorname{Aut}_{K_{s}}(A)\right)=K / \wp K$, and if $A_{d}$ corresponds to $d \in K$ and $A$ has the equation $Y^{2}+X Y=X^{3}+a X^{2}+b$ then $A_{d}$ has the equation $Y^{2}+X Y=X^{3}+(a+d) X^{2}+b$. If $\bar{K}=K\left(\wp^{-1}(d)\right)$ then $A_{d}=\mathbf{Z}_{d} \otimes_{\mathbf{z}} A$ with the obvious definition of $\mathbf{Z}_{d}$, and the same results hold as above.

If $j(A)=0$ or 1728 , then $\mathrm{Aut}_{K_{s}}(A)$ has order $4(j=1728$, char $\neq 2,3)$, $6(j=0, \operatorname{char} \neq 2,3), 12(j=0, \operatorname{char}=3)$ or $24(j=0, \operatorname{char}=2)$ and there are many more cases to consider.

Proposition 7. Assume that $A$ is a simple abelian variety (i.e. simple over $K)$. Let $s: G \rightarrow \operatorname{Aut}_{K}(A)$ be a homomorphism whose image is a finite subgroup contained in the centre $R$ of $\operatorname{End}(A)$. Then $s(G)$ is cyclic, of order $m$ say. Let $R_{i}, 0 \leqq i \leqq m-1$, be $R$ regarded as a G-module by $\sigma a=s(\sigma)^{i} a$ and let $A_{i}=R_{i} \otimes_{R} A$. Then, if $L$ is the fixed field of $H=\operatorname{ker}(s)$, there is an isogeny of degree $m^{m d} N_{L / K} A_{L} \rightarrow A_{0} \times A_{1} \times \cdots \times A_{m-1}$.

Proof. Let $\sigma_{0}$ generate $G / H$ and let $\zeta=s\left(\sigma_{0}\right)$. Then the homomorphism $\phi: R[G / H] \rightarrow \prod R_{i}$ with matrix $\left(\zeta^{i j}\right)_{0 \leqq i, j \leqq m-1}$ relative to the obvious bases has determinant $\sqrt{m^{m}}$. 
Example.1. If $A, A_{d}$ are abelian curves as in the example above, then the proposition shows there is an isogeny $N_{K / K} A \rightarrow A \times A_{d}$ of degree 4 .

2. In the situation of the proposition, $\zeta_{A_{L}}(s)=\zeta_{N_{\mathcal{L} / K} A}(s)=\prod_{i=0}^{m} \zeta_{A_{i}}(s)$. For example, suppose that $A$ has complex multiplication over $K$ by $F=R \otimes_{\mathbf{Z}} \mathbf{Q}$ and let $\rho_{x}: I_{K} \rightarrow F_{\infty}^{*}$ be as defined in $[13, \mathrm{p} .513]$. Then

$$
\begin{aligned}
& \zeta_{A_{i}}(s)=\prod_{\sigma} L\left(s, \chi_{i, \sigma}\right), \\
& \zeta_{A_{L}}(s)=\prod_{i=0}^{m} \prod_{\sigma} L\left(s, \chi_{i, \sigma}\right)
\end{aligned}
$$

where $\sigma$ runs through the embeddings $F \rightarrow \mathbf{C}$ and $\chi_{i, \sigma}$ is the composite $I_{K} \stackrel{s^{\prime} \cdot p_{\infty}}{\longrightarrow} F_{x}^{*} \stackrel{1 \otimes \sigma}{\longrightarrow} C^{*}$ (s induces, in a canonical way, a map $I_{K} \rightarrow F \rightarrow F_{\infty}$, and we have used the same letter to denote this map).

Now let $K$ be a global field of non-zero characteristic. An abelian curve $A$ over $K$ is said to be a twisted constant curve if $A \otimes_{K} K_{s}$ is constant i. e., of the form $A_{0} \otimes_{k_{s}} K_{s}$ where $k_{s}$ is the constant field of $K_{s}$. Equivalently, $A$ is a twisted constant abelian curve if $j(A)$ is in the constant field of $K$, or if $\operatorname{End}_{K}(A) \neq \mathbf{Z}$.

Theorem 2. Let $A$ be a twisted constant abelian curve over $K$ such that $j(A) \neq 0,1728$ and char $(K) \neq 2$. Then $(B-S / D)$ is true for $A$.

Proof. Since $j(A)$ belongs to the constant field of $K$, there is a constant elliptic curve $A_{0}$ over $K$ such that $j\left(A_{0}\right)=j(A)$ i.e. such that $A$ is a $K_{s} / K$ form of $A_{0}$. In fact (see the above examples) there is a quadratic extension $\bar{K}$ of $K$ such that $A$ is a $\bar{K} / K$-form of $A_{0}$. By Proposition 7, there is an isogeny of degree $4, N_{\mathrm{K} / \mathrm{K}} A_{\mathrm{K}} \rightarrow A_{0} \times A$. By [7], $(B-S / D)$ is true for $A_{0}$ and $A_{K}$, and by Theorem 1 it is true for $N_{K / K} A_{K}$. Since $(B-S / D)$ is compatible with isogenies of degree prime to the characteristic of $K$ [16] and with products, the theorem follows.

\section{§ 3. Abelian Varieties with Complex Multiplication}

$K, \bar{K}, G, A$ will be as in $\S 2$. We write $\operatorname{End}^{0}(A)=\operatorname{End}(A) \otimes_{\mathbf{Z}} \mathbf{Q}$.

Theorem 3. Let $\bar{K} / K$ be of finite degree $m$. Suppose that $\operatorname{End}_{K}^{0}(A)$ contains a commutative subalgebra $E_{\bar{K}}$ such that $\left[E_{K}: E_{K}\right]=m$ where $E_{K}=$ $\operatorname{End}_{K}^{0}(A) \cap E_{K}$. Assume that $E_{K}$ is a field. Then $N_{K / K} A_{K}$ is isogenous to $A^{m}$.

Proof. Let $\alpha_{1}, \ldots, \alpha_{m}$ be elements of $E_{R} \cap \operatorname{End}_{K}(A)$ which are linearly independent over $R=\operatorname{End}_{K}(A)$. Consider the homomorphism $\psi: A_{\mathrm{K}}^{m} \stackrel{\phi}{\longrightarrow}$ $A_{K}^{m} \stackrel{p-1}{\longrightarrow}\left(N_{K / K} A\right)_{K}$ where $\phi$ has matrix $\left(\alpha_{j}^{\sigma_{i}}\right)\left(G=\left\{\sigma_{1}, \ldots, \sigma_{m}\right\}\right)$ and $P$ is as defined in $\S 1$ (note that here $A_{\widetilde{K}}^{\sigma_{1}}$ is canonically isomorphic to $A_{R}$ ). Obviously, $\psi^{\sigma}=\psi$, and so $\psi$ defines a homomorphism $A^{m} \rightarrow N_{K / K} A$. 
Moreover the method of the proof of Proposition 6 may be used to show that $\operatorname{deg}(\psi)=\left|N_{R / \mathbf{Z}}\left(d_{S / R}\right)\right|^{d / r}$ where $r=\operatorname{rank}_{\mathbf{z}} R, S=R\left[\alpha_{1}, \ldots, \alpha_{m}\right]$, and $d_{S / R}$ is the discriminant of $S$ over $R$.

Corollary. In the situation of the theorem.

(a) $A(\bar{K}) \otimes_{\mathbf{Z}} \mathbf{Q} \approx\left(A(K) \otimes_{\mathbf{Z}} \mathbf{Q}\right)^{m}$ and so $\operatorname{rank}(A(\bar{K}))=m \operatorname{rank} A(K)$ (see also [6]).

Assume also that $K$ is a global field.

(b) $\zeta_{A \bar{K}}(s)=\zeta_{A}(s)^{m}, \xi_{A \bar{K}}(s)=\xi_{A}(s)^{m}$,

$$
N_{\bar{K} / K}\left(\mathfrak{f}\left(A_{K}\right)\right) \cdot d_{\tilde{K} / K}^{2 d}=\mathfrak{f}(A)^{m} .
$$

(c) $(B-S / D)$ is true for $A$ over $K$ if and only if it is true for $A_{\mathcal{K}}$ over $\bar{K}$.

Proof. These all follow from the results in $\S 1$.

Example. Let $A$ be an abelian curve over $\mathbf{Q}$ which has complex multiplication by $F$. Then the conjecture $(B-S / D)$ is true for $A$ over $\mathbf{Q}$ if and only if it is true for $A$ over $F$.

Remark 1 . The theorem has a partial converse. Let $\bar{K} / K$ be Galois of degree $m$ and assume that $A$ is simple and that $E_{K}=\operatorname{End}_{K}^{0}(A)$ is commutative. If $N_{\mathbb{R} / K} A$ is isogenous to $A^{m}$ then $\left[\operatorname{End}_{K}^{0}(A): \operatorname{End}_{K}^{0}(A)\right]=m$ and the isogeny is formed, as above, by taking elements of $\operatorname{End}_{K}(A)$ which form a basis for $\operatorname{End}_{K}^{0}(A)$ over $\operatorname{End}_{K}^{0}(A)$.

Indeed, if $\psi: A^{m} \rightarrow N_{K / K} A_{K}$ is the isogeny, then $\alpha=p \psi_{\mathrm{K}}: A_{\mathrm{K}}^{m} \rightarrow A_{\mathrm{K}}$ can be written $\alpha=\left(\alpha_{1}, \ldots, \alpha_{m}\right)$ with $\alpha_{i} \in \operatorname{End}_{\mathcal{K}}(A)$. Since $\psi$ is an isogeny, $p \psi_{R}=\left(\alpha_{j}^{\sigma_{j}}\right): A_{K}^{m} \rightarrow A_{R}^{m}$ is an isogeny, and hence $\operatorname{det}\left(\alpha_{j}^{\sigma_{i}}\right) \neq 0$. This implies that $\left\{\alpha_{1}, \ldots, \alpha_{m}\right\}$ is a basis for $E_{K}$ over $E_{K}$.

2. Assume that $A$ is simple over $K$ and let $E$ be the centre of $\operatorname{End}_{K_{S}}^{0}(A)$. Let $\bar{K}$ be the smallest field containing $K$ and such that $E \subset \operatorname{End}_{K}^{\delta}(A)$. Then $\bar{K}$ is a finite Galois extension of $K$ and $\bar{K}, K, A, E$ satisfy the conditions of the theorem.

Indeed, $\operatorname{Gal}\left(K_{s} / K\right)$ acts on $E \subset \operatorname{End}_{K_{s}}^{0}(A)$ and has fixed subfield $E_{K}=E \cap \operatorname{End}_{K}^{0}(A)$. Let $H$ be the subgroup of $\operatorname{Gal}\left(K_{\mathrm{s}} / K\right)$ of elements which act trivially on $E$. Then $\bar{K}$ is the fixed field of $H$, and so $[\bar{K}: K]=$ $\left(\mathrm{Gal}\left(K_{\mathrm{s}} / K\right): H\right)=\left[E: E_{K}\right]$.

We now apply the theorem to abelian varieties with complex multiplication. For the remainder of the paper, we let $A$ be an abelian variety over a number field $K$ which (over $C$ ) is of $C M$-type $(F, \Phi)$ in the sense of [14]. We shall assume always that the image of $F$ in $\operatorname{End}_{\mathbf{C}}^{0}(A)$ is stable under the action of $\mathrm{Gal}\left(K_{s} / K\right)$. This will be true when $A$ is simple over $\mathrm{C}$ (for then $F=\operatorname{End}_{\mathbf{C}}^{0}(A)$ ), when $F_{1}=F \cap \operatorname{End}_{K}^{0}(A)$ is the maximal real subfield of $F$ (for then $F=F_{1} E$ where $E$ is the centre of $\operatorname{End}_{\mathbf{C}}^{0}(A)$, and $E$ is stable under $\operatorname{Gal}\left(K_{s} / K\right)$ ), or, more generally, when $A / K$ satisfies 
the conditions of Theorem 12 of [15]. Let $\bar{K}$ be the smallest field containing $K$ such that $F \subset \operatorname{End}_{K}^{0}(A)$. Then $G=\mathrm{Gal}(\bar{K} / K)$ acts on $F$ and has fixed field $F_{K}=F \cap \operatorname{End}_{K}^{0}(A)$. $K, K, A, F$ satisfy the conditions of the theorem.

Now let $\Sigma$ be the set of embeddings $t: F \rightarrow$ C. $G$ acts on $\Sigma$ on the right. If $t \in \Sigma$ we write $\chi_{t}$ for the Grössen-character $\chi_{t}: I_{K} \rightarrow \mathbf{C}^{*}$ defined in [13, p. 513] (note that we do not require a Grössen-character to take values in the unit circle).

Lemma. $L\left(s, \chi_{t}\right)=L\left(s, \chi_{t o}\right)$ for all $\sigma \in G, t \in \Sigma$.

Proof. It is easy to see that the homomorphism $\varepsilon: I_{K} \rightarrow F^{*}$ defined in [13, Theorem 10] commutes with the action of $G$. Fix a prime $v$ of $K$. If $\gamma_{t}$ is unramified at the primes over $v$, then the factor of $L\left(s, \gamma_{t}\right)$ (resp. $\left.L\left(s, \chi_{1 \sigma}\right)\right)$ corresponding to primes over $v$ is

$$
\prod_{w \mid v} \frac{1}{1-\chi_{t}\left(i_{w}\right) N w^{-s}} \quad\left(\text { resp. } \prod_{w \mid v} \frac{1}{1-\chi_{t \sigma}\left(i_{w}\right) N w^{-s}}\right)
$$

where $i_{w}$ is the idele whose component is 1 at primes $\neq w$ and a uniformizing parameter at $w$. By definition,

$$
\begin{aligned}
\chi_{t}\left(i_{w}\right) & =t \varepsilon\left(i_{w}\right), \\
\chi_{t \sigma}\left(i_{w}\right) & =t \sigma \varepsilon\left(i_{w}\right)=t \varepsilon\left(\sigma i_{w}\right)=t \varepsilon\left(i_{\sigma w}\right) .
\end{aligned}
$$

Since $\sigma$ permutes the primes dividing $v$, this shows that the two factors are equal. If $\chi_{t}$ is ramified at one prime dividing $v$ then it is ramified at all such primes and both factors are 1.

Theorem 4. With the above notations,

Proof. Write

$$
\zeta_{A}(s)=\prod_{t \in \Sigma / G} L\left(s, \chi_{t}\right)
$$

$$
\zeta_{A}(s)_{v}=\frac{1}{P_{A, v}\left(N v^{-s}\right)} \quad \text { and } \quad L(s, \chi)_{v}=\prod_{w \mid v} \frac{1}{1-\chi\left(i_{w}\right) N w^{-s}}
$$

(or 1) for the factors of $\zeta_{\boldsymbol{A}}(s)$ and $L(s, \chi)$ corresponding to $v$.

Let $m=[\bar{K}: K]$. Then

$$
\begin{array}{rlrl}
\zeta_{A}(s)_{v}^{m} & =\zeta_{N_{\bar{K} / K} A}(s)_{v} & & (\text { Theorem 3) } \\
& =\prod_{w \mid v} \zeta_{A_{\bar{K}}}(s)_{w} & & (\text { Proposition 3) } \\
& =\prod_{t \in \Sigma} L\left(s, \chi_{t}\right)_{v} & & ([14],[13]) \\
& =\prod_{t \in \Sigma / G}\left(L\left(s, \chi_{t}\right)_{t}\right)^{m} . &
\end{array}
$$


Both $\zeta_{A}(s)_{v}$ and $\prod_{t \in \Sigma / G} L\left(s, \chi_{t}\right)_{r}$ are functions of the form $\prod \frac{1}{1-\alpha_{i} N v^{-s}}$ and it is easy to see from this that the above equation implies that $\zeta_{A}(s)_{v}=\prod_{t \in \Sigma / G} L\left(s, \chi_{t}\right)_{v}$.

Remark. 1. If we regard the $\chi_{t}$ as characters of the Weil group $\mathscr{G}_{K}$ of $\bar{K}[18]$ then it is possible to define induced characters $\chi_{t *}$ on $\mathscr{G}_{\bar{K}}$. Moreover (loc cit.) $L\left(s, \chi_{t *}\right)=L\left(s, \chi_{t}\right), \mathfrak{i}\left(\chi_{t *}\right)=\mathfrak{f}\left(\chi_{t}\right) d_{\bar{K} K}$. Thus, our results may be stated as follows: let $A / K$ satisfy the conditions as above. Then, if $\left[F: F_{K}\right]$ $=m$, there are $2 d / m$ (quasi-) characters $\chi_{i}: \mathscr{G}_{K} \rightarrow \mathbf{C}^{*}$ such that $\zeta_{A}(s)=$ $\prod_{i} L\left(s, \chi_{i}\right), \mathfrak{f}(A)=\mathfrak{f}\left(\chi_{i}\right)^{2 d / m}$.

2. If for a Grössen-character $\chi$ of $\bar{K}, L(s, \chi)$ is multiplied by appropriate factors corresponding to the conductor of $\chi$ and to the infinite primes of $\bar{K}$, then the function $A(s, \chi)$ obtained satisfies the functional equation $\Lambda(2-s, \bar{\chi})=w A(s, \chi)$ with $|w|=1$ (assuming that $\chi(i)=\chi_{0}(i)$ $|i|^{\frac{1}{2}}$ where $\chi_{0}$ is a Grössen-character which takes its values in the unit circle). Moreover, one checks that $\xi_{A_{\bar{K}}}(s)=\prod_{t \in \Sigma} \Lambda\left(s, \chi_{1}\right)$ (up to a trivial constant). Thus $A / \bar{K}$ satisfies Serre's conjecture $\left[12, C_{9}\right], \xi_{A_{\bar{K}}}(2-s)=$ $w \xi_{A \bar{K}}(s)$, with $w=1$.

After the above theorems, this result may be extended to $A / K$. In fact, one finds easily that $\xi_{A}(s)=\prod_{t \in \Sigma / G} A\left(s, \chi_{t}\right)$, from which it follows that $\xi_{A}(2-s)=w \xi_{A}(s)$, with $w= \pm 1 .\left(w= \pm 1\right.$ because $w(\chi)=w(\bar{\chi})^{-1}$, and so if $L(s, \chi)=L(s, \bar{\chi})$ then $w(\chi)=w(\bar{\chi})= \pm 1$.)

3. Theorem 4 (and the following discussion) is closely related to a result of Shimura [15, Thm. 12]. However, his conditions are apparently more complicated and he does not compute the factors of $\zeta_{A}(s)$ (and $\mathfrak{f}(A))$ corresponding to bad primes.

Perhaps it is worth clarifying the behaviour of $A$ at bad primes. If $A$ has complex multiplication defined over $K$ then, for any prime $v$ of $K$, $A$ either has good reduction or totally unipotent reduction at $v[13$, p. 504] i. e. in the notation of $\S 1$ either $\alpha_{v}(A)=d\left(\right.$ and $\left.\varepsilon_{v}(A)=0\right)$ or $\lambda_{v}(A)=d$ (and $\varepsilon_{v}(A)=2 d$ ). If, on the other hand, $A, K, \bar{K}$ are as above, and $[\bar{K}: K]$ $=m>1$ then

$$
\begin{aligned}
& \alpha_{v}(A)=\frac{1}{m} \sum_{w \mid v} f(w \mid v) \alpha_{w}\left(A_{K}\right), \\
& \mu_{v}(A)=0, \\
& \lambda_{v}(A)=\frac{1}{m} \sum_{w \mid v} f(w \mid v)\left(\operatorname{de}(w \mid v)-d+\lambda_{w}(A)\right)
\end{aligned}
$$

(see $\S 1$ ) where $e(w \mid v)$ is the ramification index of $w$ over $v$ (in $\bar{K} / K$ ) and $f(w \mid v)$ is the residue class degree. Shimura $[15$, p. 536] gives an example 
where $\operatorname{dim}(A)=3, K=\mathbf{Q}, \bar{K}=\mathbf{Q}\left(\zeta+\zeta^{-1}, \sqrt{-11}\right)$ with $\zeta$ a primitive 7 th root of $1, m=6$, and $A$ has good reduction at the unique prime $w$ of $\bar{K}$ dividing $v=7$. Then $f(w \mid v)=2$ (with $v=7$ ), and so $\alpha_{v}(A)=1, \lambda_{v}(A)=2$, and $\varepsilon_{v}(A)=4$. Thus $A$ has bad reduction at 7 but the factor of $\zeta_{A}(s)$ corresponding to 7 is $\neq 1$.

We give two final applications of Theorem 3 .

Theorem 5. Let $A / K, G, \bar{K}$ be as in the discussion preceding the lemma above.

(a) For all primes $l, \operatorname{End}_{K}(A) \otimes \mathbf{Q}_{l} \rightarrow \operatorname{End}_{H}\left(V_{l} A\right)$ is an isomorphism, where $H=\operatorname{Gal}\left(K_{s} / K\right)$.

(b) Conjecture 2 of $[17, \mathrm{p} .104]$ is true for $A$ and $i=1$ i.e. the rank of the Néron-Severi group of $A$ is equal to the order of the pole of the 2-part of the zeta function of $A$ at $s=2$.

Proof. (a) follows from the results in [14] if $A$ has all of its complex multiplications defined over $K$. Write $H_{0}=\mathrm{Gal}\left(K_{\mathrm{s}} / \bar{K}\right) \subset H$ and $A_{*}=$ $N_{K / K} A$. Then $M_{m}\left(\operatorname{End}_{K}^{0}(A)\right) \approx \operatorname{End}_{K}^{0}\left(A_{*}\right) \approx \operatorname{End}_{\mathrm{K}}^{0}\left(A_{*}\right)^{G}$. But, $\operatorname{End}_{\mathrm{K}}^{0}\left(A_{*}\right) \otimes$ $\mathbf{Q}_{l} \approx \operatorname{End}_{H_{0}}\left(\mathbf{Q}_{i}[H] \otimes_{\mathbf{Q}_{i l}\left[H_{0}\right]} V_{i}\left(A_{K}\right)\right)$ as $G$-modules, and $M_{m}\left(\operatorname{End}_{H}\left(V_{l} A\right)\right) \approx$ $\operatorname{End}_{H_{0}}\left(\mathbf{Q}_{i}[H] \otimes_{\mathbf{Q}_{i}\left[H_{0}\right]} V_{i}\left(A_{K}\right)\right)^{G}$. (b) is proved in [10] when $A$ has all of its complex multiplications defined over $K$, and the general case may be deduced similarly to the above.

\section{References}

1. Deuring, M.: Die Zetafunktion einer algebraischen Kurve vom Geschlechte Eins IV. Nachs. d. Akad. Wiss. p. 55-80. Göttingen, 1957.

2. Greenberg, M.: Schemata over local rings II. Ann. of Math. 78, 256-266 (1963).

3. Grothendieck, A.: Fondements de la géométrie algébrique (extracts du Séminaire Bourbaki, 1957-62).

4. Grothendieck, A. (with J. Dieudonné): Eléments de géométrie algébrique. Publ. Math. I.H.E.S. (1960-1967).

5. Grothendieck, A.: Modèles de Néron et monodromie. Exposé IX of S.G. A. 7, I.H. E.S., 1967-68.

6. Honda, T.: Isogenies, rational points, and sections of group varieties. Jap. Jour. of Math. 31, 84-101 (1960).

7. Milne, J.: The Tate-Šafarevič group of a constant abelian variety. Inventiones math. 6 , 91-105 (1968).

8. Mumford, D.: Abelian varieties. Oxford U. P., London (1970).

9. Ono, T.: On the Tamagawa number of algebraic tori. Ann. of Math. (2) 78, 47-72 (1963).

10. Pohlmann, H.: Algebraic cycles on abelian varieties of complex multiplication type. Ann. of Math. 88, 161-180 (1968).

11. Serre, J.-P.: Corps Locaux. Act. Sci. Ind. 1296. Paris: Hermann 1962.

12. Serre, J.-P.: Facteurs locaux des fonctions zêta des variétés algébriques (définitions et conjectures). Séminaire Delange - Pisot - Poitou, $11^{\mathrm{e}}$ année, $\mathrm{n}^{0} 19(1969 / 70)$.

13. Serre, J.-P., Tate, J.: Good reduction of abelian varieties. Ann. of Math. 88, 492-517 (1968).

14. Shimura, G., Taniyama, Y.: Complex multiplication of abelian varieties and its applications to number theory. Publ. Math. Soc. Japan, nº 6. Tokyo, 1961. 
15. Shimura, G.: On the zeta-function of an abelian variety with complex multiplication. Ann. of Math. 94, 504-533 (1971).

16. Tate, J.: On the conjecture of Birch and Swinnerton-Dyer and a geometric analog. Séminaire Bourbaki 1965/66, exposé 306.

17. Tate, J.: Algebraic cycles and poles of zeta functions. Arithmetical algebraic geometry, p. 93-110. New York: Harper and Row (1965).

18. Weil, A.: Sur la théorie du corps de classes. J. Math. Soc. Japan 3, 1-35 (1951).

19. Weil, A.: Adeles and algebraic groups. Lecture notes. Princeton: Institute for Advanced Study 1961.

\author{
J.S. Milne \\ Department of Mathematics \\ University of Michigan \\ Ann Arbor, Mich. 48104 \\ USA
}

(Received January 22,1972/May 31,1972) 